\title{
Human concentrations of uric acid scavenges adaptive and maladaptive ROS in isolated rat hearts subjected to ischemic stress
}

Neoma T Boardman ${ }^{1}$, Aleksander Tank Falck ${ }^{1}$, Trine Lund ${ }^{1}$, Xi Chu ${ }^{1}$, Montserrat MartinArmas $^{1}$, Jon V Norvik ${ }^{1,2}$, Trond G Jenssen ${ }^{2,3}$, Kirsti Ytrehus ${ }^{1}$

${ }^{1}$ Cardiovascular Research Group, Dept of Medical Biology, UiT-Arctic University of Norway, ${ }^{2}$ Metabolic and Renal Research Group, Dept of Clinical Medicine, ,UiT-Arctic University of Norway, ${ }^{3}$ Department of Organ Transplantation, Section of Nephrology, Oslo University Hospital.

Running title: Uric acid modifies ischemic injury in the heart

Keywords: uric acid, ROS, cardioprotection, ischemic pre-conditioning, pressure overload/high pressure

Corresponding author: Kirsti Ytrehus

Department of Medical Biology, UiT -The Arctic University of Norway, N- 9037

Tromsø, Norway.

Tel: +4777644781

Fax: +4777623200

E-mail: kirsti.ytrehus@uit.no

\begin{tabular}{|c|c|}
\hline Abbreviations: & \\
\hline DHE & dihydroethidium \\
\hline GSK3 $\beta$ & glycogen synthase-3 beta \\
\hline IL-6 & interleukin-6 \\
\hline mtDNA & mitochondrial DNA \\
\hline MCP-1 & monocyte chemoattractant protein-1 \\
\hline NAD & nicotinamide adenine dinucleotide \\
\hline $\mathrm{NADH}$ & nicotinamide adenine dinucleotide (reduced form) \\
\hline NO & nitric oxide \\
\hline eNOS & endothelial nitric oxide synthase \\
\hline NOX4 & NADPH oxidase 4 \\
\hline O.C.T & optimal cutting temperature compound \\
\hline qRT-PCR & $\begin{array}{l}\text { real-time quantitative reverse transcription polymerase chain } \\
\text { reaction }\end{array}$ \\
\hline SDS & sodium dodecyl sulfate \\
\hline TMRM & tetramethylrhodamine \\
\hline $\mathrm{XO}$ & Xanthine oxidase \\
\hline ROS & reactive oxygen species \\
\hline
\end{tabular}




\begin{abstract}
Uric acid is a purine degradation product but also an important antioxidant and ROS scavenger. Experimental settings that mimic myocardial ischemia-reperfusion have not included uric acid despite that it is always present in human extracellular fluid and plasma. We hypothesized that uric acid has an important role in myocardial ROS scavenging. Here, we tested the cardiac response to uric acid on infarct size following ischemia-reperfusion with and without exacerbated oxidative stress due to acute pressure overload and during preconditioning. We also examined mitochondrial respiration and ROS-induced mitochondrial permeability transition pore opening. Under exacerbated ROS stress induced by high pressure perfusion, uric acid lowered oxidative stress and reduced infarct size. In contrast, uric acid blocked cardioprotection induced by ischemic preconditioning. However, this effect was reversed by probenecid, an inhibitor of cellular uptake of uric acid. In accordance, in intact cardiomyocytes, extracellular uric acid reduced the susceptibility of mitochondria towards opening of the permeability transition pore, suggesting that uric acid may prevent ischemia-reperfusion injury due to scavenging of maladaptive ROS. Moreover, as uric acid also scavenges also adaptive ROS, this may interfere with preconditioning. Altogether, uric acid might be a confounder when translating preclinical experimental results into clinical treatment.
\end{abstract}




\section{Introduction}

In humans and other higher primates, uric acid (UA) is the end-product of purine metabolism whereas in other mammals the presence of uricase ensures its further degradation (Sautin and Johnson 2008). As such, UA is at markedly lower levels in rodent models of ischemic heart disease. UA is synthesized by xanthine oxidase (XO) that can in turn be induced by myocardial ischemia. In line with this, the extracellular release of purine metabolites characterizes oxygen shortage and ischemic cell damage (Farthing et al. 2015). Chronically, elevated serum UA is associated with increased risk of heart failure, including heart failure with preserved ejection fraction (HFpEF) (Liese et al. 1999; Norvik et al. 2017; Norvik et al. 2016; Odden et al. 2014). In addition, elevated serum UA levels may predict the risk for myocardial infarction and underlie adverse outcomes in patients with myocardial infarction undergoing percutaneous coronary intervention (Mandurino-Mirizzi et al. 2018). Reactive oxygen species (ROS) are a byproduct when XO catalyzes the reactions of hypoxanthine to xanthine and xanthine to uric acid. A number of experimental studies have elucidated a detrimental role for XOderived ROS produced by XO in cardiac ischemia (Akizuki et al. 1985; Chambers et al. 1985; Granger and Kvietys 2015; Mozaffari et al. 2011; Mozaffari et al. 2013) and in the pathology of heart failure (Jia et al. 2015; Norvik et al. 2016). In addition, previous studies have shown that high levels of UA also exacerbate oxidative stress and an inflammatory response (Gersch et al. 2009; Kanbay et al. 2013; Sautin et al. 2007).

UA functions as an antioxidant in human plasma, and is a potent scavenger of peroxynitrite in the extracellular compartment (Juhasz et al. 2011; Kuzkaya et al. 2005). Studies of UA as acute treatment have indicated this action may improve cardiac dysfunction following ischemia-reperfusion (Lee et al. 2003) however, exogenous administration of UA may also block cardioprotection induced by conditioning (Babiker et al. 2017). These differences may be dependent on intracellular uptake of UA. Despite the role for UA in scavenging ROS and although it is always present in human plasma, experimental settings to mimic myocardial ischemia-reperfusion (IR) have not, to our knowledge, included UA. This warranted further investigation of the actions of UA in IR. Thus, in the present study our aim was to investigate effects of the presence of UA, at a 
concentration comparable to what is normally found in human plasma during cardiovascular ischemic stress, in an ex-vivo model of myocardial IR. We hypothesized that UA had an important role in myocardial ROS scavenging and may also alter ROSinduced stress adaptation central to ischemic preconditioning (IPC). Thus, we tested the cardiac response to UA on infarct size following IR during exacerbated oxidative stress due to acute pressure overload, during preconditioning and in mitochondrial ROS stress. Under these conditions, we demonstrate that in the presence of UA, ischemia-reperfusion injury was reduced, as well as oxidative stress. However, cardioprotection induced by preconditioning was blocked in the presence of UA.

\section{Methods}

\section{Animals}

Rats were treated in accordance with the Guide for the Care and Use of Laboratory Animals ( $8^{\text {th }}$ edition, National Academies Press) and the guidelines on accommodation and care of animals formulated by the European Convention for the Protection of Vertebrate Animals for Experimental and Other Scientific purposes. All animal experiments in the present study were approved by the Norwegian National Animal Research Authority (FDU ID 2929), as well as the local authority (Department of Comparative Medicine, UiT-Arctic University of Norway) of the National Animal Research Authority in Norway .

Male Wistar rats (Charles River, Germany) were given ad libitum access to standard chow and water and housed in a room with 12-h:12-h light-dark cycle at $21^{\circ} \mathrm{C}$. On the day of experiments, they were heparinized (200 IU i.p) and anesthetized (pentobarbitone sodium $50 \mathrm{mg} / \mathrm{kg}$ i.p). The heart was excised and prepared for either perfusion, or cell or mitochondrial isolation.

Isolated heart perfusions. All hearts were Langendorff-perfused using Krebs-Henseleit bicarbonate buffer (KHB) containing $2.1 \mathrm{mM} \mathrm{Ca}^{++}$and $11 \mathrm{mM}$ glucose, equilibrated with $95 \% \mathrm{O}_{2}-5 \% \mathrm{CO}_{2}$ at $37^{\circ} \mathrm{C}$. Standard perfusion pressure was set to $100 \mathrm{~cm} \mathrm{H}_{2} \mathrm{O}(73.6 \mathrm{~mm}$ 
Hg). For experiments investigating low versus high perfusion pressure, perfusion pressure was set at 80 (LP) and $200 \mathrm{~cm} \mathrm{H}_{2} \mathrm{O}$ (HP) (58.8 and $147 \mathrm{~mm} \mathrm{Hg}$ ), respectively. A water-filled latex balloon, connected to a pressure transducer, was inserted into the left ventricle via the left atrium, and inflated to set a left ventricular end-diastolic pressure (LVDevP) between 0-10 mmHg as baseline, which was thereafter left unchanged.

Experimental protocols in isolated hearts. All hearts underwent 20 minutes stabilization followed by either 25 minutes regional ischemia (coronary artery ligation) or 30 minutes of global ischemia and 120 minutes of reperfusion (Figure 1). Ischemic preconditioning (IPC) was induced by one cycle of 5 minutes global ischemia followed by 5 minutes of reperfusion, prior to the ischemia and reperfusion protocol (I/R). In one set of experiments, uric acid (100 or $500 \mu \mathrm{M}$ ) was administered in the perfusion buffer for a total of 10 minutes prior to IPC and regional I/R. To test whether cellular uric acid uptake altered the infarct-sparing effect of IPC, we added probenecid ( $1 \mu \mathrm{M})$, an inhibitor of organic anion transporter and pannexin channel (Silverman et al. 2008), to the perfusion buffer. Probenecid was added to the buffer 5 minutes prior to the introduction of uric acid and followed by global I/R. In another set of experiments, isolated perfused hearts were subjected to regional ischemia and reperfusion during LP or HP-perfusion. Uric acid (500 $\mu \mathrm{M}$ ) or allopurinol (1 mM) was added to the perfusion buffer for assessment of infarct size and functional recovery under LP or HP-perfusion.

Infarct size determination. For regional infarct studies, a silk suture was placed around the main branch of the left coronary artery and ischemia was induced by tightening the suture using a snare. Following reperfusion the coronary artery was again ligated and Evans Blue dye was infused via the aorta to demarcate the myocardial risk zone. For infarct size measurements, the hearts were weighed, frozen at $-20^{\circ}$, and cut into $2 \mathrm{~mm}$ thick slices. The slices were incubated for 15 minutes in 1\% triphenyl-tetrazolium chloride (TTC; Sigma-Aldrich) in phosphate buffer ( $\mathrm{pH} 7.4,37^{\circ} \mathrm{C}$ ), immersed in $10 \%$ formaldehyde to enhance the contrast between viable and necrotic tissue, and thereafter the tissue slices were scanned to obtain a digital image. Infarct size was expressed as the 
ratio of infarcted area (lacking staining with TTC) to risk zones or to the ventricle area when using global ischemia using ImageJ software (NIH).

Western blot of heart tissue. To investigate whether perfusion pressure altered preischemic activation of kinase cascades related to cell survival, left ventricle tissue was rapidly frozen in liquid nitrogen and tested for phosphorylation of Akt, p44/42 MAPK, GSK3 $\beta$. Heart tissue was homogenized in $10 \mathrm{ml}$ ice-cold PBS buffer containing $1 \%$ Igepal CA-630, 0.1 \% SDS, 0.5\% Sodium Deoxycholate, 5mM EDTA, 2mM EGTA, 100 ul Phosphatase inhibitor 2, 100 ul Phosphatase inhibitor 3, 10 ul beta-Mercaptoethanol, 1 tablet EDTA-free Complete Protease inhibitor cocktail (all supplied by Sigma). Thereafter the homogenate was centrifuged at $14000 \mathrm{~g}$ for $10 \mathrm{~min}$ at $4^{\circ} \mathrm{C}$ and protein content was measured (Bradford, BioRad protein assay). Supernatant samples (30 $\mu \mathrm{g}$ protein) underwent SDS-page electrophoresis using a standard Laemmli loading buffer (Criterion anyKD, Biora). The following antibodies from Cell Signaling Technology

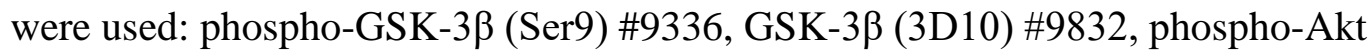
(Ser473) \#9271, Akt \#9272, p44/42 MAPK (ERK1/2) \#9102, phospho p44/42 MAPK (Thr202/Tyr204) \#9101, and actin \#A2066 (all polyclonal except the GSK-3ß (3D10) antibody).

Detection of intracellular ROS production in heart tissue by dihydroethidium (DHE) staining. DHE reacts with superoxide radicals to form ethidium bromide, which in turn intercalates with DNA to provide fluorescence as a marker of ROS, especially superoxide radical generation (Camara et al. 2004; Kevin et al. 2003). DHE was used to investigate if ROS production at reperfusion in HP-perfused hearts was influenced by allopurinol or uric acid treatment. DHE (10 $\mathrm{mM}$ ) was added to the perfusion-buffer for 3 minutes prior to global ischemia and during the first 5 minutes of reperfusion such that the ROS indicator was present at the same time as allopurinol or uric acid. Following experiments, left ventricular tissue was embedded in O.C.T compound, frozen in cooled isopentane and stored at $-70^{\circ} \mathrm{C}$. The samples were cryo-sectioned and imaged under an 
epifluorescence microscope. Between 18-20 images from each heart was obtained for quantification using Image J software.

Mitochondrial permeability transition ( $\mathrm{mPTP}$ ) in cardiomyocytes. To examine if the extracellular concentration of uric acid affected the susceptibility of mitochondria in intact cardiomyocytes towards opening of the permeability transition pore when exposed to ROS, we incubated tetra methyl rhodamine methyl ester (TMRM) loaded cardiomyocytes with different concentrations of uric acid. Hypoxanthine, the precursor of XO derived uric acid, was used as control. Cardiomyocytes were isolated using collagenase (Worthington type 2, Lakewood, NJ 08701, US) based on the method described by Myrmel et al. 1991 (Myrmel et al. 1991). Freshly isolated cells were resuspended in HEPES buffer containing TMRM (Molecular Probes) $125 \mathrm{nM}$ for $60 \mathrm{~min}$ at room temperature. Isolated TMRM loaded cardiomyocytes were then placed in an open glass bottomed chamber, allowed time to settle and superfused with TMRM containing HEPES with and without the addition of uric acid $(0,10,50,100500 \mu \mathrm{M})$ or hypoxanthine $(0,10,50,100,500 \mu \mathrm{M})$. Laser confocal microscopy (Zeiss $510 \mathrm{LSM}$, excitation at $543 \mathrm{~nm}$ and emission above 560) was used in line scan mode, and focused on a row of mitochondria to obtain a timed series of loss of TMRM from selected mitochondrial compartments (Juhaszova et al. 2004). By this technique, the effect of uric acid on ROS dependent mPTP transition was quantified as time to mPTP transition.

Mitochondrial respiration. To investigate if uric acid present in myocardial cells or taken up from the cell-surroundings influenced mitochondrial respiration, mitochondria were isolated from rat hearts and respiration examined in 6 paired experiments with and without uric acid (500 uM) present. Isolation of mitochondria was performed using the procedure described by Palmer et al. (Palmer et al. 1977) with slight modifications. Briefly, heart tissue was weighed and placed in a buffer containing (in mM): $100 \mathrm{KCl}$; 50 MOPS; 1 EGTA; $5 \mathrm{MgSO}_{4}$; 1 ATP and pH 7.4. The tissue was minced with scissors, and homogenized with a Polytron for 7 seconds followed by 10 min incubation with $5 \mathrm{mg} / \mathrm{mL}$ 
trypsin at $4{ }^{\circ} \mathrm{C}$. Buffer with $2 \mathrm{mg} / \mathrm{ml}$ BSA was then added to the tissue supernatant and homogenized with two strokes of a Potter-Elvehjem homogenizer (Glas-Col, Terre Haute, IN, USA) and centrifuged at $590 \mathrm{~g}$ for $10 \mathrm{~min}$ at $4^{\circ} \mathrm{C}$. The supernatant was filtered through a nylon net (NITEX $300 \mathrm{~lm}$; Yulee, FL, USA) and centrifuged at $3000 \mathrm{~g}$ for $10 \mathrm{~min}$ at $4^{\circ} \mathrm{C}$. The mitochondrial pellet was resuspended in buffer $\left(4^{\circ} \mathrm{C}\right)$ and centrifuged again at $3000 \mathrm{~g}$ for $10 \mathrm{~min}$. The new pellet was now resuspended in buffer containing (in mM):100 KCl, 0.5 EGTA, 50 MOPS, pH 7.4, and centrifuged at $3000 \mathrm{~g}$ for $10 \mathrm{~min}$ at $4^{\circ} \mathrm{C}$. The final pellets were resuspended and left on ice for $30 \mathrm{~min}$ to stabilize the membranes. Measurement of mitochondrial oxygen consumption was performed in duplicate by Oxygraph-2k (Oroboros, Austria) using a respiration medium containing in mM:100 KCl; 50 MOPS; 1 EGTA; $5 \mathrm{KH}_{2} \mathrm{PO}_{4}$ and BSA 1 mg/mL, pH = 7.4 in a closed $2 \mathrm{~mL}$ chamber at $37^{\circ} \mathrm{C}$. Uric acid $500 \mu \mathrm{M}$ was added to one of the two parallel chambers of the oxygraph at the start of each experiment. Glutamate $(10 \mathrm{mM})$ and malate $(1 \mathrm{mM})$ were used to assess complex I driven respiration. After obtaining a stable state 2 respiration, ADP was added to a final concentration of $200 \mu \mathrm{M}$ (state 3). Thereafter oxygen consumption was recorded after the depletion of ADP (state 4) and ADP added to final concentration $2 \mathrm{mM}$ to obtain maximal ADP-stimulated respiration. Finally, leak supported respiration was assessed by adding oligomycin $(4 \mu \mathrm{g} / \mathrm{mL})$ to inhibit the ATPsynthase. All values were normalized to citrate synthase activity measured spectrophotometrically using the enzymatic conversion of 5,5'-dithiobis (2-nitrobenzoic acid) to thionitrobenzoic acid which has an intense absorption at $412 \mathrm{nM}$ (Holloszy et al. 1970; Kuznetsov et al. 2002).

Statistics. All results are presented as mean \pm SEM. Statistical comparisons were performed with SigmaPlot version 14.0 (Systat software) using one-way ANOVA with the Holm-Sidak post-hoc test or Student's t-test where appropriate, where differences of $\mathrm{p}<0.05$ were considered significant. For the one-way ANOVA analysis, the F-value and $\mathrm{p}$-value are also presented in the figure text. 


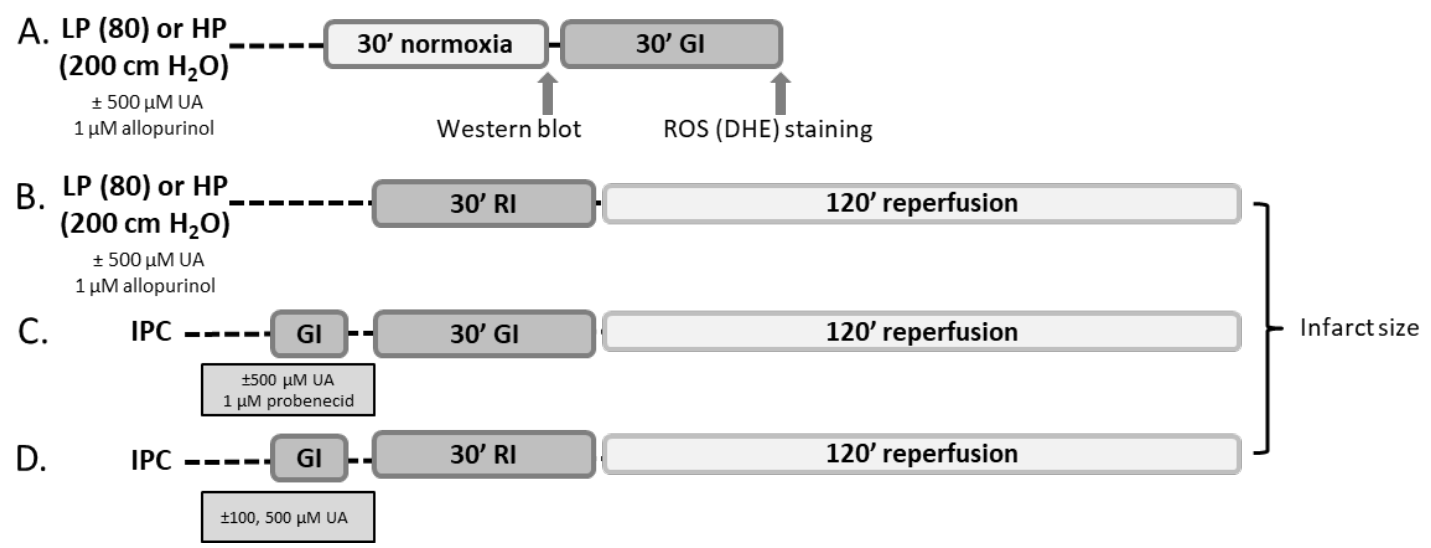

Figure 1. Ischemia-reperfusion (IR) protocols in isolated rat hearts with exacerbated ROS stress or ischemic preconditioning (IPC). To induce oxidative stress, hearts were perfused with high pressure (HP) or low pressure (LP), and underwent regional ischemia (RI) followed by reperfusion and measurement of infarct size. IPC was induced by 1 cycle of 5 minutes global ischemia (GI) following by 5 minutes reperfusion prior to either GI or RI.

\section{Results}

\section{Uric acid and allopurinol protected high-pressure perfused hearts against ischemia reperfusion injury.}

In agreement with other studies (Mozaffari et al. 2011; Mozaffari and Schaffer 2008), pressure overload resulted in significantly higher \% infarct/risk zone (53.5 \pm 2.2 versus $30.6 \pm 4.0 \%$ for HP vs. LP, respectively) (Fig.2A) and caused oxidative stress (Figure 5). To assess ROS-scavenging effects during HP-perfusion we used $500 \mu \mathrm{M}$ concentration of UA, as $100 \mu \mathrm{M}$ did not inhibit cardioprotection by IPC (Figure 3A). UA reduced infarct size in HP- perfused isolated hearts to $41.1 \pm 3.9 \%$. The presence of $1 \mathrm{mM}$ allopurinol in the buffer also reduced infarct size in pressure-overloaded hearts to $37.0 \pm 4.7$. None of the treatments reduced infarct size in LP-perfused hearts (data not shown).

Pressure overload resulted in increased cardiac work as shown by increased LV developed pressure (LVDevP) and dp/dt max as compared to LP-perfused hearts (Figure 2B). Neither uric acid nor allopurinol altered developed pressure. Perfusion with allopurinol in pressure overloaded hearts resulted in an increased $\mathrm{dP} / \mathrm{dt}$ max both at 
baseline and at the end of the ischemia-reperfusion protocol above HP alone (5397.7 \pm 333 vs. $7180.2 \pm 768.7$ and $2551.9 \pm 146.2$ vs. $3541-7 \pm 488.0$, for HP-perfused hearts with and without allopurinol, respectively), which was not observed with UA.

A.

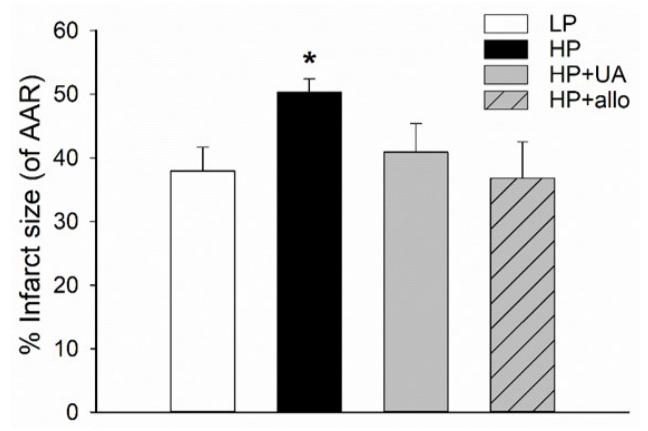

B.
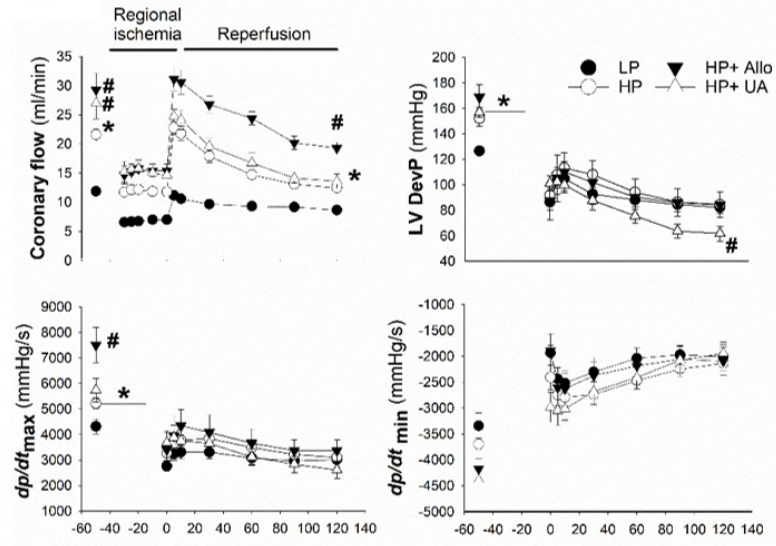

Time (min)

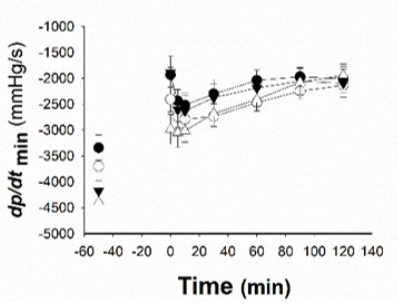

Figure 2. A) Both uric acid (UA) and allopurinol prevented high-pressure perfusion induced increase in infarct size following regional ischemia reperfusion. Mean \pm SEM, $n=5-9$ for all groups. ( $F=2.798, p<0.045)$ and $p<0.05$ vs. low pressure (LP) - perfusion. B) High-pressure (HP) perfusion increased baseline cardiac contractile function and coronary flow. Only allopurinol maintained increased coronary flow following ischemia-reperfusion. Left ventricle developed pressure (LVDevP) was lower during reperfusion in hearts treated with uric acid (UA) and remained similar within the other groups. Mean \pm SEM, $n=5-10$. Coronary flow: At baseline (F=37.103, $\mathrm{p}<0.001),{ }^{*} \mathrm{p}<0.05$ vs. low-pressure (LP), and \# $<0.05$ vs. HP; and at time 120 minutes ( $\mathrm{F}=6.77, \mathrm{p}<0.002),{ }^{*} \mathrm{p}<0.05$ vs. LP and and $\# \mathrm{p}<0.05$ vs. HP. LVDevP: At baseline ( $\mathrm{F}=6.77, \mathrm{p}<0.002),{ }^{*} \mathrm{p}<0.05$ vs. LP; and at time $120 \mathrm{~min}(\mathrm{~F}=3.67, \mathrm{p}<0.026), \# \mathrm{p}<0.05$ vs. HP. Dp/dt $t_{\max }:(\mathrm{F}=11.04, \mathrm{p}<0.001),{ }^{*} \mathrm{p}<0.05$ vs. LP and $(\mathrm{F}=11.041, \mathrm{p}<0.001), \# \mathrm{p}<0.05$ vs. HP. No significant differences in $d p / d t_{\min }$ at baseline or during reperfusion.

\section{Uric acid blocked cardioprotection by ischemic preconditioning (IPC)}

The addition of either 100 or $1000 \mu \mathrm{M}$ UA alone did not alter the \% infarct/risk zone (data not shown). However, UA inhibits ischemic preconditioning (IPC) -induced cardioprotection in isolated rat hearts subjected to IPC under standard perfusion pressure prior to ischemia reperfusion. At low concentrations $(100 \mu \mathrm{M}) \mathrm{UA}$ did not prevent IPC- 
induced reduction in infarct size. At elevated levels $(500 \mu \mathrm{M})$, there was no effect of IPC on infarct size (Figure 3A) suggesting that UA may scavenge ROS and prevent ROS signalling which is essential in IPC cardioprotection. The infarct lowering effect of IPC was observed in the presence of allopurinol, which is known to inhibit ROS production by xanthine oxidase. In another set of experiments, probenecid was added to the perfusion buffer to limit cellular uptake of UA (Figure 3B). Probenecid did not alter infarct size on its own; however, it did maintain the infarct sparing effects of IPC in the presence of UA. This suggests that probenecid diminished the intracellular scavenging ability of UA and did not disturb the ROS-related signalling central in IPC-induced protection.

A.

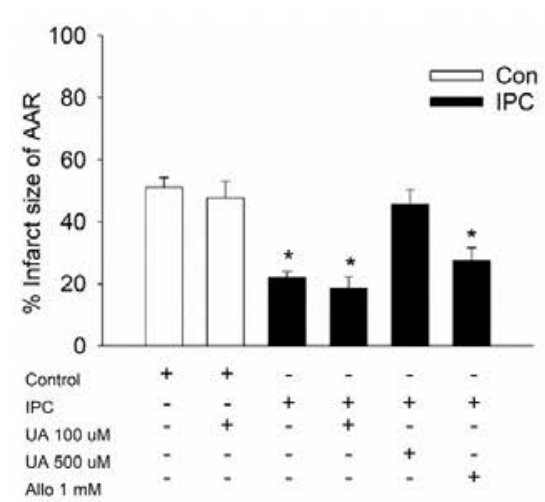

B.

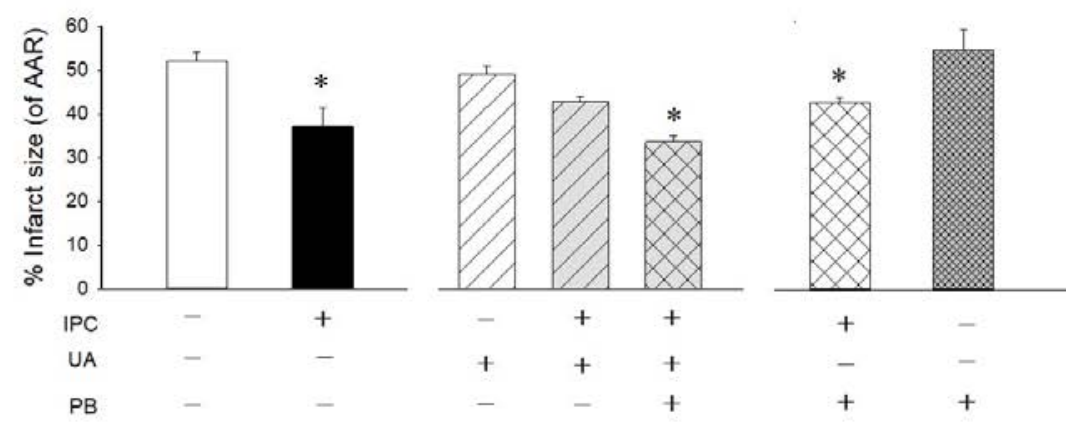

Figure 3. A) Uric acid (UA) but not allopurinol inhibits ischemic preconditioning (IPC) -induced cardioprotection in isolated rat hearts subjected to IPC prior to regional ischemia reperfusion. Mean \pm SEM, $n=5-8 .(F=18.9, p<0.001),{ }^{*} \mathrm{p}<0.05$ vs. control (without IPC). B) Blocking cellular uric acid (UA) uptake by probenecid maintains ischemic preconditioning (IPC) -induced cardioprotection in isolated rat hearts subjected to IPC prior to global ischemia reperfusion. Mean \pm SEM, $\mathrm{n}=5-8$ for all groups. ( $\mathrm{F}=8.36, \mathrm{p}<0.001),{ }^{*} \mathrm{p}<0.05$ vs. control (no IPC).

\section{Uric acid and allopurinol reduced ROS content but did not alter survival protein kinases}

Under normoxic conditions, HP-perfusion elevated phosphorylation of protein kinases GSK-3 $\beta$, ERK and AKT as compared to hearts that underwent LP perfusion (Figure 4). Neither UA nor allopurinol blocked this increase. 
DHE-reactive ROS in tissue samples harvested at reperfusion following 25 min global ischemia was higher in HP-perfused hearts compared to LP. Both Allopurinol and UA blocked the HP-perfusion related ROS increase (Figure 5).
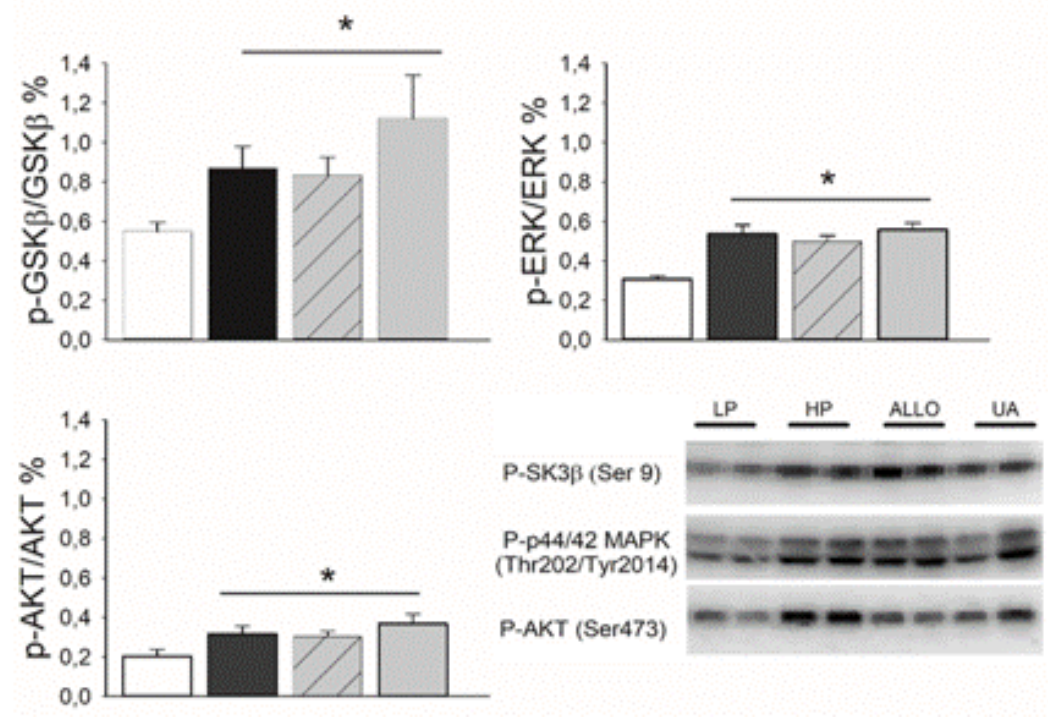

Figure 4. High pressure (HP) perfusion induced phosphorylation of cell survival kinases as compared to low pressure (LP) and was not altered by treatment with uric acid (UA) or allopurinol. The relative balance between phosphorylated and total GSK3 $\beta$, PKB/AKT and ERK, and their representative western blots, in hearts harvested prior to ischemia in groups LP, HP, HP + Allo, LP + UA. Mean \pm SEM, n=5-6. GSK: (F=2.746, $\mathrm{p}=0.07),{ }^{*} \mathrm{p}<0.05$ vs. LP; ERK: ( $\mathrm{F}=9.956, \mathrm{p}<0.001),{ }^{*} \mathrm{p}<0.05$ vs LP; AKT: $(\mathrm{F}=2.73, \mathrm{p}=0.08),{ }^{*} \mathrm{p}<0.05$ vs. LP.
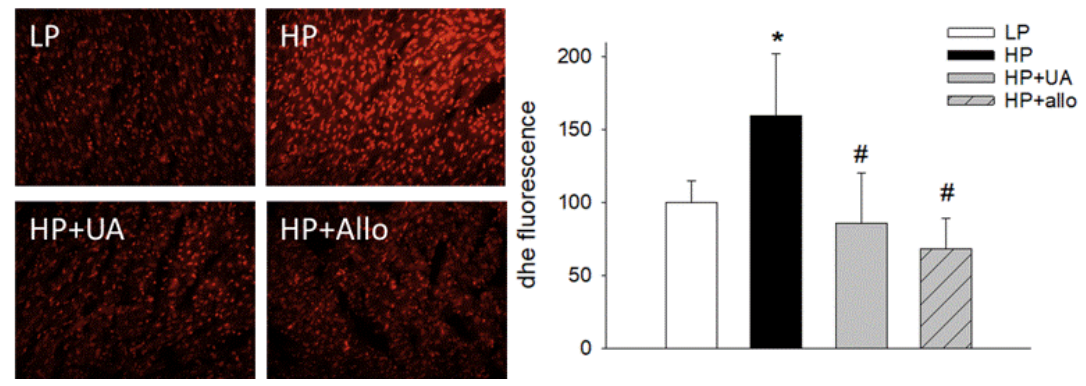

Figure 5. High pressure (HP) perfusion increased ROS content measured by dihydroethidium (DHE) fluorescence intensity but this was reduced in the presence of uric acid (UA) and allopurinol. Mean \pm SEM, $\mathrm{n}=5$ for all groups. ( $\mathrm{F}=7.77, \mathrm{p}<0.002),{ }^{*} \mathrm{p}<0.05$ vs low pressure (LP) and $\# \mathrm{p}<0.05$ vs. HP. 


\section{Uric acid protected mitochondria in isolated cardiomyocytes against permeability transition but reduced OXPHOS in isolated heart mitochondria}

Addition of UA ( $500 \mu \mathrm{M})$ to the superfusion chamber of isolated cardiomyocytes protected mitochondria as seen by the significantly increased time to mitochondrial permeability transition pore opening (mPTP) (Figure 6A). Increased time to pore opening was evident also at lower and higher concentrations of UA. However, comparable experiments with the UA precursor, purine hypoxanthine, did not reveal significant protection against pore opening.

In isolated mitochondria, the addition of UA $(500 \mu \mathrm{M})$ resulted in a slight reduction in respiratory control ratio (RCR; state 3/state 4) which may suggest reduced mitochondrial function (Figure 6B). Complex I driven respiration (state 3) at low and saturating levels of ADP was significantly reduced compared to that observed in controls $(12.6 \%$ and 15.2\%, respectively). Oligomycin-induced leak respiration (State 4) was unchanged suggesting that UA did not increase mitochondrial uncoupling (Brand and Nicholls 2011).

A.

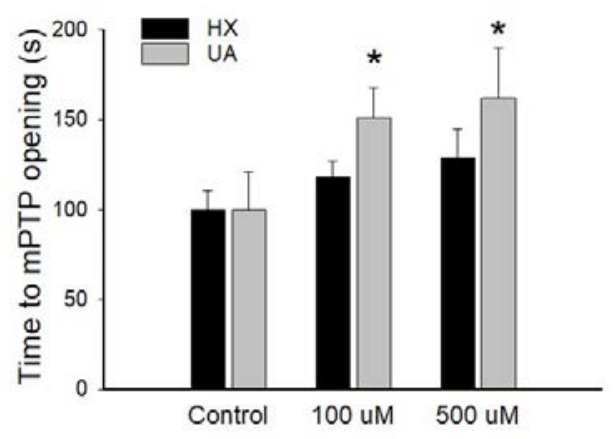

B.

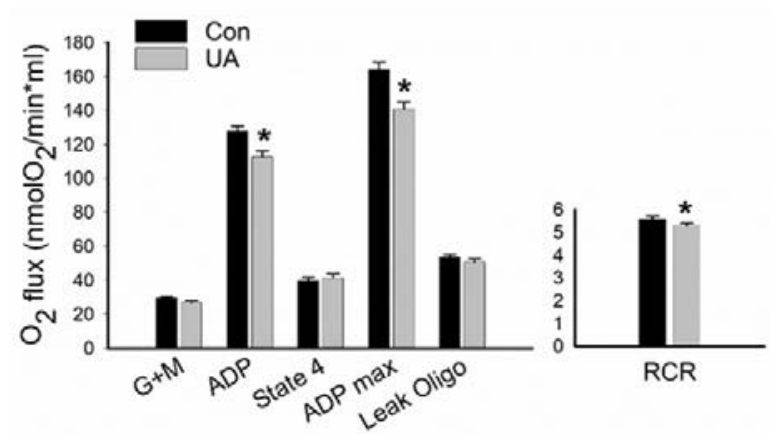

Figure 6. A) Time to opening of the mitochondrial permeability transition pore (mPTP). This was examined in tetramethylrhodamin (TMRM)-loaded cardiomyocytes with either hypoxanthine (HX) or uric acid (UA) in indicated concentrations added to the buffer. Results are \% of mean value in corresponding control groups (97.8 \pm 10.5 and 108.5 \pm 22.6 sec). Mean \pm SEM, $\mathrm{n}=5-7$. ( $\mathrm{F}=4.24, \mathrm{p}<0.008),{ }^{*} \mathrm{p}<0.05$ vs. control. $\mathrm{B}$ ) Uric acid lowered mitochondrial oxidative phosphorylation at sub-maximal and maximal ADP concentrations (ADP and ADP max, respectively) and lowered the respiratory control ratio (RCR). Oxygen flux was normalized to citrate synthase levels. Mean \pm SEM, $n=5-7 .{ }^{*} \mathrm{p}<0.05$ vs. control, using Student's t-test. 


\section{Discussion}

Although uric acid (UA) is associated with pathological detriment in heart disease, it also functions as an antioxidant in human plasma, and is a potent scavenger of peroxynitrite in the extracellular compartment (Juhasz et al. 2011; Kuzkaya et al. 2005). The present study confirms that presence of UA, at human concentrations, modulates the response to ischemia. UA 1) reduces ex-vivo ischemia-reperfusion injury when combined with increased perfusion pressure, 2) blocks cardioprotection by ischemic preconditioning (IPC), and 3) protects against mitochondrial pore opening in isolated myocardial cells. Thus, this study confirms that concentrations corresponding to those normally present in human blood could have an infarct-sparing effect.

Cardioprotection by IPC is dependent on signaling ROS, where one cycle of five minutes of ischemia followed by five minutes of reperfusion triggers an adaptation (Sovershaev et al. 2006; Starkopf et al. 1998). Here, peroxynitrite is considered to play a central role (Laude et al. 2002). However, limitation of reperfusion injury by different cardio-protective treatments such as UA or XO inhibition, is also closely associated with reduction in ROS (George et al. 2006; Pagliaro and Penna 2015). Thus, our results are in agreement with the proposed scavenging effects of acute elevations of UA seen in previous studies (Babiker et al. 2017; Lee et al. 2003; Mozaffari et al. 2011).Pressure overload (increased perfusion pressure) at reperfusion after prolonged ischemia increases reperfusion injury in experimental studies (Gunnes et al. 1990; Mozaffari et al. 2011; Mozaffari and Schaffer 2008). The present findings partly confirms earlier studies demonstrating that abrupt restoration of perfusion pressure after global ischemia might

add to injury if pressure is elevated, and supports the notion that blood pressure control is important during reperfusion therapy (Lindal et al. 1990; Pedrinelli et al. 2012;

Reinstadler et al. 2016). Interestingly, we did not find UA or allopurinol to reduce the elevated developed pressure in HP-perfused hearts. In contrast, the present study demonstrates that perfusion with allopurinol in pressure overloaded hearts resulted in an increased $\mathrm{dP} / \mathrm{dt}$ max both at baseline and at the end of the ischemia-reperfusion protocol, which was not observed with UA. The reason for this was not examined in detail, but could be related to differences in pressure overload induced-ROS levels between these 
studies, and the dose dependency of contractile performance during ROS exposure of isolated rat hearts (Hegstad et al. 1997; Xie et al. 1998).

Consistent with previous reports that the activity of XO and ROS are central mechanisms contributing to aggravated ischemia-reperfusion induced cell death due to elevated perfusion pressure (Mozaffari et al. 2011), both UA and allopurinol reduced infarct size in isolated rat hearts in the present study. There are several sources of ROS in the heart, most of them proposed to be active in relation to ischemia-reperfusion injury and vascular dysfunction (Granger and Kvietys 2015). The combination of high oxygen demand/oxidative stress, and NO stimulation in the vessels by pressure overload, can promote peroxynitrite formation, and trigger XO activity (Mozaffari et al. 2011). HPperfusion induced increase in oxygen demand may also contribute to a lower cardiac efficiency, which has previously been related to increased susceptibility to ischemiareperfusion injury (Lund et al. 2015). However, reduced cardiac efficiency is unlikely to be the primary cause of HP-induced increase in cell death as allopurinol treatment also reduced cell death following IR, despite increased contractile work. HP-perfusion itself also significantly affected the degree of phosphorylation of various cell survival related signaling kinases, Akt, ERK and GSK3 $\beta$, which are linked to cardioprotection by preconditioning (Hausenloy et al. 2017; Johansen et al. 2011; Juhaszova et al. 2004). In contrast to these studies, increased phosphorylation of AKT, ERK and GSK3 $\beta$ was associated with markedly increased infarct size. Moreover, IPC-induced cardioprotection was observed in HP-perfused hearts. UA and allopurinol did not affect phosphorylation of these kinases prior to ischemia.

Under physiological conditions as well as in our perfusion solutions, UA exists as organic anion urate. Probenecid is a highly lipid soluble benzoic acid derivative used to inhibit urate reuptake in renal tubular cells. Interestingly, probenecid has been described as both a pannexin channel inhibitor as well as a urate re-uptake inhibitor (George et al. 2006; Silverman et al. 2008). In the present study, probenecid prevented UA from blocking IPC-induced cardioprotection and supports that effective UA uptake does occur in the heart. Probenecid by itself did not modify infarct size or inhibit ischemic preconditioning. However, more studies are required to fully understand UA uptake mechanisms in cardiac cells. 
The mitochondrial response to UA was also tested. UA delayed opening of the mPTP following acute exposure of isolated cardiomyocytes to intracellular ROS. This observation is most likely due to the ROS scavenger effect of UA and is in accordance with the ability of UA to reduce reperfusion injury and ischemic preconditioning under the conditions described in the present study. Few studies have looked at the direct effect of UA on mitochondrial function. There is a sustained interest in regulation of the mitochondrial membrane potential, which in turn, drives the $\mathrm{F}_{1} \mathrm{~F}_{0}$-ATPase activity: However, how this is coupled to ATP degradation products like UA is not well clarified (Kristal et al. 1999). When cardiac mitochondria were incubated with UA, oxidative phosphorylation decreased slightly, but significantly. Purine metabolism differs between organs and cells and relates to ATP consumption, turnover of mtDNA and nuclear DNA as well as cellular enzymatic makeup, resulting in variable intracellular levels of UA. Differences in uptake from the extracellular space further adds to this complexity. Recently, Wang et al. proposed a strong coupling of ATP production and consumption via the mito-flash phenomenon and $\mathrm{F}_{1} \mathrm{~F}_{0}$-ATPase (Gong et al. 2015; Wang et al. 2017).

Limitations. Cells and tissue from rodents are not adapted to extracellular UA levels used in the present study, which are comparable to levels in human extracellular fluid. On one hand, the present study indicates that adding UA in amounts present in humans reveals an antioxidant effect of UA that is difficult to demonstrate in clinical studies. On the other hand, the study does not explain the significant association between plasma UA level and cardiovascular pathophysiology. The question of whether UA is a casual, compensatory, or coincidental factor in cardiovascular medicine remains unanswered, and needs to be solved on a human background (Carluccio et al. 2018; So and Thorens 2010) as well as in uricase-deficient experimental animals. So far, the number of studies with human derived cells and tissue comparing exposure to different UA concentrations are limited. Interestingly, a human study of individuals with loss of function mutations in UA transporter URAT1, which led to hypouricemia, described concomitant significant endothelial dysfunction (Sugihara et al. 2015).

In conclusion, we have demonstrated that under experimental conditions in isolated perfused hearts, cardiomyocytes and mitochondria, the presence or absence of 
UA in concentrations equivalent with human plasma concentrations, modulated the response to cardiac ischemia. UA behaved like a highly ambiguous agent, protecting against I/R injury under certain conditions and maintaining mitochondrial integrity, but also blocked protection by endogenous ischemic preconditioning of the heart and tended to reduce mitochondrial respiration. As UA is continuously present in human plasma, it may reduce the effect of conditioning treatments to enhance cardioprotection in human studies. Potential interference with UA should be tested when translating experimentally promising cardioprotective interventions towards clinical application, such as ischemicpreconditioning.

\section{Acknowledgements:}

We acknowledge the late Prof. Ingrid Toft who helped conceive this study.

\section{Author contributions:}

N.T.B, A.T.F, T.L, X.C, M.M-A, J.V.N, and K.Y conceived and planned experiments. N.T.B, A.T.F, T.L, X.C, and M.M-A carried out the experiments and analyzed the data. N.T.B, A.T.F, T.L, X.C, M.M-A, J.V.N, T.G.J and K.Y contributed to interpretation of results. N.T.B and K.Y designed the figures and wrote the manuscript with input from all authors.

Competing interests: none 


\section{References}

Akizuki, S., Yoshida, S., Chambers, D.E., Eddy, L.J., Parmley, L.F., Yellon, D.M., and Downey, J.M. 1985. Infarct size limitation by the xanthine oxidase inhibitor, allopurinol, in closed-chest dogs with small infarcts. Cardiovasc Res 19(11): 686-692.

Babiker, F.A., Al-Jarallah, A., and Joseph, S. 2017. Understanding pacing postconditioningmediated cardiac protection: a role of oxidative stress and a synergistic effect of adenosine. $\mathrm{J}$ Physiol Biochem 73(2): 175-185. doi: 10.1007/s13105-016-0535-z.

Brand, M.D., and Nicholls, D.G. 2011. Assessing mitochondrial dysfunction in cells. Biochem J 435(2): 297-312. doi: 10.1042/BJ20110162.

Camara, A.K., Riess, M.L., Kevin, L.G., Novalija, E., and Stowe, D.F. 2004. Hypothermia augments reactive oxygen species detected in the guinea pig isolated perfused heart. Am $\mathrm{J}$ Physiol Heart Circ Physiol 286(4): H1289-1299. doi: 10.1152/ajpheart.00811.2003.

Carluccio, E., Coiro, S., and Ambrosio, G. 2018. Unraveling the relationship between serum uric acid levels and cardiovascular risk. Int J Cardiol 253: 174-175. doi: 10.1016/j.ijcard.2017.11.035. Chambers, D.E., Parks, D.A., Patterson, G., Roy, R., McCord, J.M., Yoshida, S., Parmley, L.F., and Downey, J.M. 1985. Xanthine oxidase as a source of free radical damage in myocardial ischemia. J Mol Cell Cardiol 17(2): 145-152.

Farthing, D.E., Farthing, C.A., and Xi, L. 2015. Inosine and hypoxanthine as novel biomarkers for cardiac ischemia: from bench to point-of-care. Exp Biol Med (Maywood) 240(6): 821-831. doi: $10.1177 / 1535370215584931$.

George, J., Carr, E., Davies, J., Belch, J.J., and Struthers, A. 2006. High-dose allopurinol improves endothelial function by profoundly reducing vascular oxidative stress and not by lowering uric acid. Circulation 114(23): 2508-2516. doi:

10.1161/CIRCULATIONAHA.106.651117.

Gersch, C., Palii, S.P., Imaram, W., Kim, K.M., Karumanchi, S.A., Angerhofer, A., Johnson, R.J., and Henderson, G.N. 2009. Reactions of peroxynitrite with uric acid: formation of reactive intermediates, alkylated products and triuret, and in vivo production of triuret under conditions of oxidative stress. Nucleosides Nucleotides Nucleic Acids 28(2): 118-149. doi: 10.1080/15257770902736400.

Gong, G., Liu, X., Zhang, H., Sheu, S.S., and Wang, W. 2015. Mitochondrial flash as a novel biomarker of mitochondrial respiration in the heart. Am J Physiol Heart Circ Physiol 309(7): H1166-1177. doi: 10.1152/ajpheart.00462.2015.

Granger, D.N., and Kvietys, P.R. 2015. Reperfusion injury and reactive oxygen species: The evolution of a concept. Redox Biol 6: 524-551. doi: 10.1016/j.redox.2015.08.020. 
Gunnes, S., Ytrehus, K., and Sorlie, D. 1990. Effects of initial reperfusion temperature and pressure after prolonged cardioplegic ischemic arrest. A metabolic and functional study in rat hearts. Scand J Thorac Cardiovasc Surg 24(2): 135-139.

Hausenloy, D.J., Garcia-Dorado, D., Botker, H.E., Davidson, S.M., Downey, J., Engel, F.B., Jennings, R., Lecour, S., Leor, J., Madonna, R., Ovize, M., Perrino, C., Prunier, F., Schulz, R., Sluijter, J.P.G., Van Laake, L.W., Vinten-Johansen, J., Yellon, D.M., Ytrehus, K., Heusch, G., and Ferdinandy, P. 2017. Novel targets and future strategies for acute cardioprotection: Position Paper of the European Society of Cardiology Working Group on Cellular Biology of the Heart. Cardiovasc Res 113(6): 564-585. doi: 10.1093/cvr/cvx049.

Hegstad, A.C., Antonsen, O.H., and Ytrehus, K. 1997. Low concentrations of hydrogen peroxide improve post-ischaemic metabolic and functional recovery in isolated perfused rat hearts. J Mol Cell Cardiol 29(10): 2779-2787. doi: 10.1006/jmcc.1997.0513.

Holloszy, J.O., Oscai, L.B., Don, I.J., and Mole, P.A. 1970. Mitochondrial citric acid cycle and related enzymes: adaptive response to exercise. Biochem Biophys Res Commun 40(6): 13681373.

Jia, G., Habibi, J., Bostick, B.P., Ma, L., DeMarco, V.G., Aroor, A.R., Hayden, M.R., WhaleyConnell, A.T., and Sowers, J.R. 2015. Uric acid promotes left ventricular diastolic dysfunction in mice fed a Western diet. Hypertension 65(3): 531-539. doi:

10.1161/HYPERTENSIONAHA.114.04737.

Johansen, D., Sanden, E., Hagve, M., Chu, X., Sundset, R., and Ytrehus, K. 2011. Heptanol triggers cardioprotection via mitochondrial mechanisms and mitochondrial potassium channel opening in rat hearts. Acta Physiol (Oxf) 201(4): 435-444. doi: 10.1111/j.1748-

1716.2010.02221.x.

Juhasz, L., Kiss, A., Nyeso, E., Kovacs, M., Seprenyi, G., Kaszaki, J., and Vegh, A. 2011. Is there a trigger role of peroxynitrite in the anti-arrhythmic effect of ischaemic preconditioning and peroxynitrite infusion? Eur J Pharmacol 667(1-3): 306-313. doi: 10.1016/j.ejphar.2011.06.010. Juhaszova, M., Zorov, D.B., Kim, S.H., Pepe, S., Fu, Q., Fishbein, K.W., Ziman, B.D., Wang, S., Ytrehus, K., Antos, C.L., Olson, E.N., and Sollott, S.J. 2004. Glycogen synthase kinase-3beta mediates convergence of protection signaling to inhibit the mitochondrial permeability transition pore. J Clin Invest 113(11): 1535-1549. doi: 10.1172/JCI19906.

Kanbay, M., Segal, M., Afsar, B., Kang, D.H., Rodriguez-Iturbe, B., and Johnson, R.J. 2013. The role of uric acid in the pathogenesis of human cardiovascular disease. Heart 99(11): 759-766. doi: 10.1136/heartjnl-2012-302535. 
Kevin, L.G., Camara, A.K., Riess, M.L., Novalija, E., and Stowe, D.F. 2003. Ischemic preconditioning alters real-time measure of $\mathrm{O} 2$ radicals in intact hearts with ischemia and reperfusion. Am J Physiol Heart Circ Physiol 284(2): H566-574. doi:

10.1152/ajpheart.00711.2002.

Kristal, B.S., Vigneau-Callahan, K.E., Moskowitz, A.J., and Matson, W.R. 1999. Purine catabolism: links to mitochondrial respiration and antioxidant defenses? Arch Biochem Biophys 370(1): 22-33. doi: 10.1006/abbi.1999.1387.

Kuzkaya, N., Weissmann, N., Harrison, D.G., and Dikalov, S. 2005. Interactions of peroxynitrite with uric acid in the presence of ascorbate and thiols: implications for uncoupling endothelial nitric oxide synthase. Biochem Pharmacol 70(3): 343-354. doi: 10.1016/j.bcp.2005.05.009. Kuznetsov, A.V., Strobl, D., Ruttmann, E., Konigsrainer, A., Margreiter, R., and Gnaiger, E. 2002. Evaluation of mitochondrial respiratory function in small biopsies of liver. Anal Biochem 305(2): 186-194. doi: 10.1006/abio.2002.5658.

Laude, K., Thuillez, C., and Richard, V. 2002. Peroxynitrite triggers a delayed resistance of coronary endothelial cells against ischemia-reperfusion injury. Am J Physiol Heart Circ Physiol 283(4): H1418-1423. doi: 10.1152/ajpheart.00375.2002.

Lee, W.H., Gounarides, J.S., Roos, E.S., and Wolin, M.S. 2003. Influence of peroxynitrite on energy metabolism and cardiac function in a rat ischemia-reperfusion model. Am J Physiol Heart Circ Physiol 285(4): H1385-1395. doi: 10.1152/ajpheart.00808.2002.

Liese, A.D., Hense, H.W., Lowel, H., Doring, A., Tietze, M., and Keil, U. 1999. Association of serum uric acid with all-cause and cardiovascular disease mortality and incident myocardial infarction in the MONICA Augsburg cohort. World Health Organization Monitoring Trends and Determinants in Cardiovascular Diseases. Epidemiology 10(4): 391-397.

Lindal, S., Gunnes, S., Ytrehus, K., Straume, B.K., Jorgensen, L., and Sorlie, D. 1990.

Amelioration of reperfusion injury following hypothermic, ischemic cardioplegia in isolated, infarcted rat hearts. Eur J Cardiothorac Surg 4(1): 33-39.

Lund, J., Hafstad, A.D., Boardman, N.T., Rossvoll, L., Rolim, N.P., Ahmed, M.S., Florholmen, G., Attramadal, H., Wisloff, U., Larsen, T.S., and Aasum, E. 2015. Exercise training promotes cardioprotection through oxygen-sparing action in high fat-fed mice. Am.J.Physiol Heart Circ.Physiol 308(8): H823-H829. doi: 10.1152/ajpheart.00734.2014.

Mandurino-Mirizzi, A., Crimi, G., Raineri, C., Pica, S., Ruffinazzi, M., Gianni, U., Repetto, A., Ferlini, M., Marinoni, B., Leonardi, S., De Servi, S., Oltrona Visconti, L., De Ferrari, G.M., and Ferrario, M. 2018. Elevated serum uric acid affects myocardial reperfusion and infarct size in patients with ST-segment elevation myocardial infarction undergoing primary percutaneous 
coronary intervention. J Cardiovasc Med (Hagerstown) 19(5): 240-246. doi:

10.2459/JCM.0000000000000634.

Mozaffari, M.S., Baban, B., Liu, J.Y., Abebe, W., Sullivan, J.C., and El-Marakby, A. 2011.

Mitochondrial complex I and $\mathrm{NAD}(\mathrm{P}) \mathrm{H}$ oxidase are major sources of exacerbated oxidative stress in pressure-overloaded ischemic-reperfused hearts. Basic Res Cardiol 106(2): 287-297. doi: 10.1007/s00395-011-0150-7.

Mozaffari, M.S., Liu, J.Y., Abebe, W., and Baban, B. 2013. Mechanisms of load dependency of myocardial ischemia reperfusion injury. Am J Cardiovasc Dis 3(4): 180-196.

Mozaffari, M.S., and Schaffer, S.W. 2008. Effect of pressure overload on cardioprotection of mitochondrial KATP channels and GSK-3beta: interaction with the MPT pore. Am J Hypertens 21(5): 570-575. doi: 10.1038/ajh.2008.25.

Myrmel, T., Forsdahl, K., Sager, G., and Larsen, T.S. 1991. Regulation of lipolysis in normoxic and hypoxic rat myocytes. J Mol Cell Cardiol 23(2): 207-215.

Norvik, J.V., Schirmer, H., Ytrehus, K., Storhaug, H.M., Jenssen, T.G., Eriksen, B.O., Mathiesen, E.B., Lochen, M.L., Wilsgaard, T., and Solbu, M.D. 2017. Uric acid predicts mortality and ischaemic stroke in subjects with diastolic dysfunction: the Tromso Study 1994-2013. ESC Heart Fail 4(2): 154-161. doi: 10.1002/ehf2.12134.

Norvik, J.V., Storhaug, H.M., Ytrehus, K., Jenssen, T.G., Zykova, S.N., Eriksen, B.O., and Solbu, M.D. 2016. Overweight modifies the longitudinal association between uric acid and some components of the metabolic syndrome: The Tromso Study. BMC Cardiovasc Disord 16: 85. doi: 10.1186/s12872-016-0265-8.

Odden, M.C., Amadu, A.R., Smit, E., Lo, L., and Peralta, C.A. 2014. Uric acid levels, kidney function, and cardiovascular mortality in US adults: National Health and Nutrition Examination Survey (NHANES) 1988-1994 and 1999-2002. Am J Kidney Dis 64(4): 550-557. doi: 10.1053/j.ajkd.2014.04.024.

Pagliaro, P., and Penna, C. 2015. Redox signalling and cardioprotection: translatability and mechanism. Br J Pharmacol 172(8): 1974-1995. doi: 10.1111/bph.12975.

Palmer, J.W., Tandler, B., and Hoppel, C.L. 1977. Biochemical properties of subsarcolemmal and interfibrillar mitochondria isolated from rat cardiac muscle. J Biol Chem 252(23): 8731-8739.

Pedrinelli, R., Ballo, P., Fiorentini, C., Denti, S., Galderisi, M., Ganau, A., Germano, G., Innelli, P., Paini, A., Perlini, S., Salvetti, M., Zaca, V., and Gruppo di Studio Ipertensione e Cuore, S.I.d.C. 2012. Hypertension and acute myocardial infarction: an overview. J Cardiovasc Med (Hagerstown) 13(3): 194-202. doi: 10.2459/JCM.0b013e3283511ee2. 
Reinstadler, S.J., Stiermaier, T., Eitel, C., Saad, M., Metzler, B., de Waha, S., Fuernau, G., Desch, S., Thiele, H., and Eitel, I. 2016. Antecedent hypertension and myocardial injury in patients with reperfused ST-elevation myocardial infarction. J Cardiovasc Magn Reson 18(1): 80. doi:

10.1186/s12968-016-0299-1.

Sautin, Y.Y., and Johnson, R.J. 2008. Uric acid: the oxidant-antioxidant paradox. Nucleosides

Nucleotides Nucleic Acids 27(6): 608-619. doi: 10.1080/15257770802138558.

Sautin, Y.Y., Nakagawa, T., Zharikov, S., and Johnson, R.J. 2007. Adverse effects of the classic antioxidant uric acid in adipocytes: NADPH oxidase-mediated oxidative/nitrosative stress. Am J Physiol Cell Physiol 293(2): C584-596. doi: 10.1152/ajpcell.00600.2006.

Silverman, W., Locovei, S., and Dahl, G. 2008. Probenecid, a gout remedy, inhibits pannexin 1 channels. Am J Physiol Cell Physiol 295(3): C761-767. doi: 10.1152/ajpcell.00227.2008.

So, A., and Thorens, B. 2010. Uric acid transport and disease. J Clin Invest 120(6): 1791-1799. doi: 10.1172/JCI42344.

Sovershaev, M.A., Egorina, E.M., Andreasen, T.V., Jonassen, A.K., and Ytrehus, K. 2006.

Preconditioning by 17beta-estradiol in isolated rat heart depends on PI3-K/PKB pathway, PKC, and ROS. Am J Physiol Heart Circ Physiol 291(4): H1554-1562. doi:

10.1152/ajpheart.01171.2005.

Starkopf, J., Andreasen, T.V., Bugge, E., and Ytrehus, K. 1998. Lipid peroxidation, arachidonic acid and products of the lipoxygenase pathway in ischaemic preconditioning of rat heart.

Cardiovasc Res 37(1): 66-75.

Sugihara, S., Hisatome, I., Kuwabara, M., Niwa, K., Maharani, N., Kato, M., Ogino, K., Hamada, T., Ninomiya, H., Higashi, Y., Ichida, K., and Yamamoto, K. 2015. Depletion of Uric Acid Due to SLC22A12 (URAT1) Loss-of-Function Mutation Causes Endothelial Dysfunction in Hypouricemia. Circ J 79(5): 1125-1132. doi: 10.1253/circj.CJ-14-1267.

Wang, X., Zhang, X., Wu, D., Huang, Z., Hou, T., Jian, C., Yu, P., Lu, F., Zhang, R., Sun, T., Li, J., Qi, W., Wang, Y., Gao, F., and Cheng, H. 2017. Mitochondrial flashes regulate ATP homeostasis in the heart. Elife 6. doi: 10.7554/eLife.23908.

Xie, Y.W., Kaminski, P.M., and Wolin, M.S. 1998. Inhibition of rat cardiac muscle contraction and mitochondrial respiration by endogenous peroxynitrite formation during posthypoxic reoxygenation. Circ Res 82(8): 891-897. 